\title{
Predicting Educational Background using Text Mining
}

\author{
Rense Corten*, Shiva Nadit, and Laurence Frank ${ }^{\dagger}$ \\ This version: March 16, 2021
}

\begin{abstract}
We examine to what extent educational background can be inferred from written text, assuming that educational levels are associated with the style of writing and use of language. Using a large public dataset of almost 60000 dating profiles, containing written text for each profile, we look for a methodology to measure author style. We focus on education and essays fields in each profile from which we try to identify relevant features of written text that reveal the level of education of authors behind texts. Using different types of extracted features, we explore the level of education within three approaches: (i) classifying the level of education to elementary or higher education using lexical features; (ii) using Linguistic Inquiry and Word Count (LIWC) features; (iii) combining LIWC features and lexical features. For classification, we rely on regularized logistic regression. The joint model which uses both lexical and LIWC features predicts the education level better than other text representation models, but the contribution of LIWC is marginal. Our results may not only be useful in the context of the platform economy and online markets, also more generally to researchers who need to rely on written text as an indicator of educational background.
\end{abstract}

Keywords: Computational linguistics, Machine learning, social class, text mining, trust

\section{INTRODUCTION}

The emergence of the internet and the accompanying digitization of social life has led to an unprecedented accumulation of data on social and economic interactions. Among many other types of data, this includes large amounts of text data produced in social interactions. This is relevant for social science from at least two perspectives. On the one hand, such text data, as far as they are available for social science research, allow for new ways to study social processes (Evans and Aceves 2016; Molina and Garib 2019). At the same time, one could also argue that the role of written text has become more prominent in day-to-day social and economic interactions. For example, texting has largely replaced calling by telephone (Sherna 2012), social media play an important role in the spreading of news and information (Gottfried and Shearer 2016), and more generally, social interactions via the internet largely (although not exclusively) take the form of written text produced by users.

A corollary of this observation is that the usage and command of language by individuals in written text can be hypothesized to have important impacts on social outcomes. As a motivating example, consider economic interactions in the so-called peer-to-peer platform economy, on online platforms such as Ebay, Airbnb or Craigslist. On such platforms, individual users (rather than businesses) who are strangers to each other conduct economic transactions, and typically, trust plays an important role in these interactions (Botsman and Rogers 2011; Corten 2019; ter Huurne, Ronteltap, et al. 2017). At the same time, participants crucially and increasingly rely on revealing personal information such as profile pictures but also written text for the formation of trust (Schor et al. 2016). That is, potential providers

\footnotetext{
* Corresponding author. Department of Sociology/ICS, Faculty of Social and Behavioural Sciences, Utrecht University. Address: Padualaan 14, 3584 CH, Utrecht, the Netherlands. E-mail: r.corten@uu.nl

+ Department of Methodology and Statistics, Faculty of Social and Behavioural Sciences, Utrecht University
} 
of goods or services try to paint a picture of themselves as competent and trustworthy, and much of this takes the form of written text on profile descriptions, messages exchanged between users, etc.

Sociological theory and some empirical research provide reasons to suspect that in such a context, the usage and command of language by individual users may impact the extent to which they are trusted by other users and more generally, be perceived as attractive exchange partners. For instance, Coleman (1990) suggests that individuals with high socioeconomic status are more likely to be trusted, because they tend to be in positions where they are more visible and opportunistic behavior is more likely to lead to a loss of reputation (also see Ridgeway 2014; Sauder, Lynn, and Podolny 2012). In addition, highstatus individuals may be perceived as more competent and more trustworthy because they are socialized as such during their upbringing and formal education, or conversely, because they would not have achieved a high-status position if they weren't competent and trustworthy (Keijzer and Corten 2017). Regardless of whether such perceptions are true, if they are held by other users, they would imply that providing credible signals of a high socioeconomic status would allow one to appear as more trustworthy, which in itself is a valuable asset in the platform economy (and as such a form of social capital; Coleman 1990). Arguably, linguistic cues could serve as such signals, also in the sense that the usage of language can be seen as a form of cultural capital, that is, a skillset that allows one to identify as a member of the higher socioeconomic strata (Bourdieu 1991). Both qualitative and quantitative empirical research indeed suggest that high-status actors are more likely to be trusted (Keijzer and Corten 2017; but also see Piff et al. 2012 for an opposing view), and that linguistic cues play a role in this process (ter Huurne, Moons, et al. 2017; Schor et al. 2016).

The argumentation sketched above suggests that the increasing reliance on written text in digital contexts, in combination with increasing emphasis on personal presentations in contexts such as the emerging platform economy, has the potential to produce new forms of social inequality, in that those with sufficient cultural capital in form of a certain command of language are able to convert this to social capital in the form of being trusted. This type of cumulative advantage (DiPrete and Eirich 2006) in the platform economy would be "new" in the sense the regular economy, perceptions of trustworthiness are more likely to rely on formal qualifications, proven competence and social embeddedness rather than on personal characteristics. However, it is also obvious that this argumentation relies on number of strong assumptions and leaves many questions open. For instance, to what extent do actors in contexts such as the platform economy actually respond to linguistic cues, and if so, which cues are these? To what extent are such cues indeed measurably correlated with socioeconomic background, and how can we measure this in empirical data?

The current paper takes a first step in exploring such questions by studying the link between userproduced text and social background. More specifically, we explore to what extent it is possible to estimate social background of the author from written text using a text mining approach, in an empirical context where the social background of the author of the text is known to the researcher. Social background in this case is defined in terms of the highest level of formal education achieved, as intuitively this is most important dimension of social background in influencing the use of language. The empirical context studied is online dating, which arguably is somewhat removed from the platform economy context that serves as the motivating example (although trust issues certainly play a role in online dating as well), but has the crucial advantage of providing both text data and sufficiently detailed information on social background (level of education, in this case).

In addition to contributing to such substantive questions about the role of user-written text in social interactions, studying the relation between written text and social background may also be useful from a methodological perspective. While digital footprints data often provide rich information on behavior that can be observed unobtrusively, such data are often also "shallow" in the sense that they provide relatively little information on the social and demographic backgrounds of users, such as level of 
education, ethnicity, and age (Salganik 2018). Survey research, on the other hand, tends to include questions on such background characteristics almost by default, and much research as well as theory in the social sciences relies heavily on such information, which sometimes limits the usefulness digital footprint data in studying "main stream" social science questions. Robust methods to measure social background from user-written text could therefore provide a valuable alternative for measuring such variables when more direct measures are not available, similar to the estimation of ethnicity from names (Hofstra and de Schipper 2018).

\section{RELATED WORK}

Earlier research has developed methods to estimate other aspects of social background than level of education from written text, in particular demographic traits such as ethnicity, gender and age (Burger et al. 2011; Nguyen et al. 2013; Nguyen, Smith, and Rose 2011), but also geographic location (Eisenstein et al. 2010; Rao et al. 2010) or more behavioral or attitudinal traits such as political orientation (Rao et al. 2010).

Substantively, our work differs from much of the existing literature in two ways. First, while much of the existing research focuses on social media and Twitter in particular, we study text from online dating profiles. Text from profiles can be expected to differ from tweets in the sense that while tweets are typically very short texts composed more or less "on the fly", on a large variation of topics, and in a largely informal conversational context, profile texts are, besides being considerably longer, more formal, and more specifically aimed at self-description, arguably also more "strategic" in the sense that they are more deliberate written, aim to put the author in a positive light and are aimed at bring positive payoffs to the author.

Second, while much of the previous literature aims at estimating demographic characteristics from text that are to a large extent "ascribed", we focus on an attribute (level of education) that is "achieved" (c.f. Blau and Duncan 1967), that is associated with social prestige. Also from this perspective, we can expect that authors have incentives to signal their level of education deliberately through their usage of language. At the same time, language also plays an important role in the achievement of a certain level of education. On the one hand, the command of language is obviously to a certain extent learned through education. On the other hand, cultural capital theory claims that language as learned at home and as a signal of social class is also instrumental in the achievement of a high level of education (Bourdieu 1973).

Methodologically, our use of longer profile texts rather than tweets also implies that not only have more but also more complex text available. This allows us, to some extent, to look into more "intricate" psychological and linguistic features of the text, and compare the predictive value of such features to more simplistic approaches such as simply counting words (i.e., bag-of-words).

A further methodological advantage of our data source over much of the existing literature, especially over studies relying on tweets, is that we have a considerable amount of social background information available as reported by the users themselves. On Twitter, users may provide limited amounts of information about themselves in their profile description, but this information is not very structured and many users simply do not provide this information. As a consequence, in estimating social or demographic characteristics of users, most previous studies have no "ground truth" available to verify their methods (e.g., Burger et al. 2011). In response, some rely on manual annotation which is both labor intensive and error-prone (Nguyen et al. 2013; Rao et al. 2010). Even if this is doable for (relatively) straightforward demographic traits such as gender, it is much more for socially constructed traits such as social class of socio-economic status. In contrast, online dating services are specifically designed to inform users about potential partners, and therefore collect a lot of information about these users. 
Perhaps closest to our approach is the work by Nguyen et al. (2013), who use Twitter data to predict age from (Dutch) tweets. Based on only unigrams, they are able to predict age, age category and life stage with considerable accuracy. In particular the method of classifying age category, using logistic regression, closely resembles our approach. Besides the data source, the langue studied, and the dependent variable, the key difference with our study is that they need to rely on human annotators for a "ground truth". In contrast, we have self-reported level of education directly available in our data. In addition, we have larger volumes of text available per author and overall a somewhat larger sample of authors, which in principle should result in a higher accuracy of predictions. At the same time, it is ex ante not obvious how the accuracy of prediction of a "attained trait" such as level of education should compare to that of an "exogenous" trait such as age.

\section{DATA}

A study on the relationship between language use in user-generated text and social background requires data that provides both samples of such text and direct measures of social background. While online platform markets serve as the motivating example for such research, such platforms do typically not provide direct measures of the social backgrounds of their users. Indeed, the fact that they do not is precisely why linguistic cues may become relevant. Instead, for this paper, we rely on a data source of a different nature, namely profiles from an online dating platform. Since the social backgrounds of potential partners tend to be very relevant to users, online dating platforms typically ask users to provide many details about their background, including their level of education, which is the focus of our study. In addition, users are typically asked to describe themselves in their own words, which provides us with samples of written text.

More specifically, we use data obtained from the online dating platform OK Cupid, which were made available for research- and teaching purposes with consent from the platform (Kim and Escobedo-Land 2015). OK Cupid (OKC) is a US-based online dating service established in 2004 with more than 50 million users worldwide (Zuckerman 2020). The service is free to join (although fees may be charged for specific features) and matches users to potential romantic partners based on the information that users provide. The available data set contains user profile data on 59,946 San Francisco OkC users, which were scraped and published with consent by the platform.

\section{Data processing}

In terms of user-produced text, the dataset includes the answers to 10 questions posed to all users in which they are asked to describe themselves (E.g., "my self summary", "On a typical Friday night I am..."; see Kim and Escobedo-Land 2015 for details). Since we are mostly interested in the linguistic aspects of the text rather than the specific content of these answers, we merge these 10 columns into one text for each user.

Level of education is reported by users in an open text field (e.g., "graduated from high school", "law school", or "space camp"). We manually recoded these strings to educational categories using the International Standard Classification of Education (UNESCO 2012). However, since a number of these categories contain no or very few cases, we collapse the educational categories into a binary variable indicating a "lower" (below Bachelor level) or "higher" (Bachelor and up) level of education. This results in $22 \%$ of the cases in the lower category. This binary variable serves as the outcome variable in a classification problem where the users are classified as lower or higher educated based on their selfwritten profiles. Before the self-written profiles can be used as explanatory variables, the texts first have to be transformed into numerical values. 
Preprocessing of text and bag-of-words representation

A standard approach to making text numerical and analysable is the bag-of-words approach (cf. Eisenstein 2019). The text documents are transformed into a vector with counts of words. This approach is called bag-of-words because the representation of the text only contains information about the frequency of the single words, and does not include information about the order of the words in the context of the sentence and the rest of the text. Grammatical and other linguistic aspects of the text are not included.

Figure 1 illustrates the bag-of-words transformation process where a sentence is transformed into a vector with the frequencies of each word. The first step is tokenization, which means splitting each profile text into separate words based on the whitespaces and punctuations in the documents. In this preprocessing step, we also removed unnecessary tokens in the user profile texts, such as HTML tags and stop words (examples are: "a", "the", "me"). Removing stop words helps to retain the words that have maximum significance and context. Other commonly used preprocessing steps are stemming (keeping only the linguistic stem of the words) and removing punctuations, capitalization, contractions and misspelled words. We did not apply these preprocessing steps because we believe that the way that users use the words, punctuations, capitalization, contractions and misspelled words reflects the writing style of users and might be related to their level of education.

After tokenization, the next step is building a vocabulary of all the words that appear in any of the profile documents and number them in alphabetical order. The result is a matrix where the rows represent the users and where the columns represent the entire set of distinct words (tokens) in all the user profile texts. The columns are binary $(0,1)$ features, where 1 indicates that the word is present in the profile text for a distinct user.

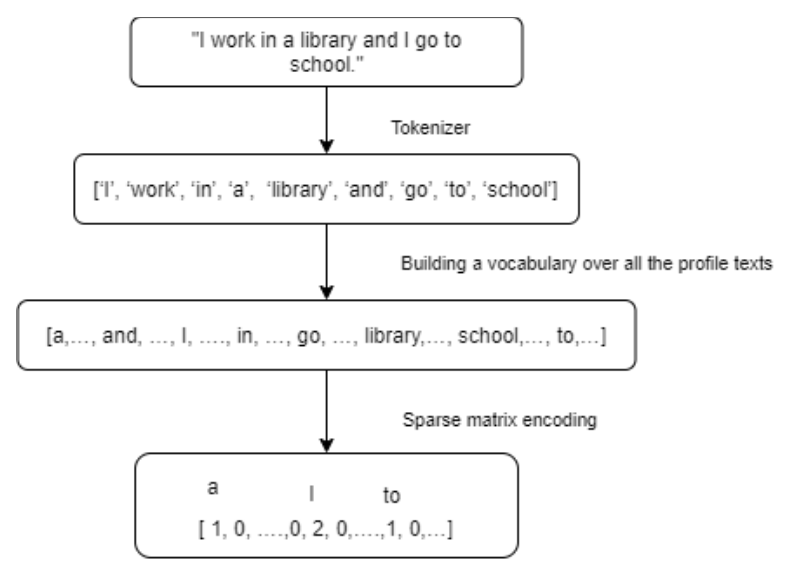

Figure 1. The bag-of-words transformation process

\section{Enriching the bag-of-words representation with linguistic and stylistic features}

Although the simple bag-of-words feature matrix can be effective in text mining classification problems, both common intuition and cultural capital theory suggest that social background may manifest itself in more complex features of language than simply the use of single words (i.e., vocabulary). To capture the context of the words, we used the count of pairs of words (bigrams) that appear next to each other in addition to single-word features (unigrams). A further way to enrich the bag-of-words representation is to look for more complex linguistic features that represent differences in content and style. To capture such features, we rely on the method of Linguistic Inquiry and Word Count (LIWC; Pennebaker 
et al. 2015). LIWC is a text analysis method that calculates the percentage of the words in a given text that fall into one or more of 90 linguistic, psychological and topical categories. According to the authors, "the words we use in daily life reflect who we are and the social relationships we are in" (Tausczik and Pennebaker 2010:25). Accordingly, LIWC was developed with the aim of automatically detecting psychologically meaningful categories of words, based on a number of dictionaries. In past research, LIWC has been successfully applied to identify emotion, intentions, features of social relationship and hierarchies, and differences between social categories in text (see Tausczik and Pennebaker 2010 for a review). LIWC thus lends itself naturally for the aims of this paper. We here use LIWC2015 v1.6 (Pennebaker et al. 2015) extracting linguistic features from profile texts. The output of LIWC is a vector with 94 features for each profile text.

\section{METHODS}

Based on the features extracted from the user profile texts as explained in the previous section, the following three different text representation models were used to predict education level: Model 1BGW contains the lexical features (unigrams and bigrams). Model 2- LWC contains the linguistic and stylistic features extracted by LIWC. Model 3- BLWC: in this model the lexical features are combined with the linguistic and stylistic features from the LIWC method. We use three classification methods were: Naive Bayes, regularized logistic regression and the neural network classifier LSTM.

The Naive Bayes classifier is often used in text classification problems because it can deal with large feature spaces (cf. Jurafsky and Martin 2008). The Naive Bayes model estimates the probability that the outcome (education level of the user) is one of the two levels, given the set of features $X$ that apply to the profile text written by the user. The assumption that the features are independent given the class $y \in Y$ dramatically simplifies the computational evaluation of the loss function:

$$
\hat{y}=\operatorname{argmin} \sum_{y \in Y} L(\hat{y}, y) P[y \mid X]
$$

Despite that the assumption of conditional independence of the features is generally not true, the Naive Bayes classifier performs surprisingly well in text classification (Jurafsky and Martin 2008).

In natural language processing, logistic regression is a very often used classifier. Given the very large number of features, we used regularized (L2) logistic regression to prevent overfitting select a subset of relevant features (cf. Hastie, Tibshirani, and Friedman 2009). L2-logistic regression regularizes the coefficient estimates towards zero by applying a constraint on the sum of the coefficients. The least squares weights $\beta_{0}, \beta_{1}, \ldots, \beta_{p}$ for the features are estimated by the following loss function:

$$
\hat{\beta}=\operatorname{argmax}_{\beta}\left[\sum_{i=1}^{n} \log P\left(y^{(i)} \mid x^{(i)}\right)\right]-\alpha \sum_{j=1}^{m} \beta_{j}^{2}
$$

The parameter $\alpha>=0$ is a complexity parameter that controls the amount of regularization (shrinkage): the larger the value of $\alpha$, the greater the amount of shrinkage.

The third classifier is the neural network classifier LSTM, which and can be viewed as a series of logistic regression classifiers stacked on top of each other. A recurrent neural network (RNN) is a generalization of the standard feed forward neural network with internal memory that is ideal for modeling sequential data. A RNN model receives an input at each timestep, updates its hidden state, 
and makes a prediction. By using recurrent connections, information can cycle inside these networks (Hochreiter and Schmidhuber 1997).

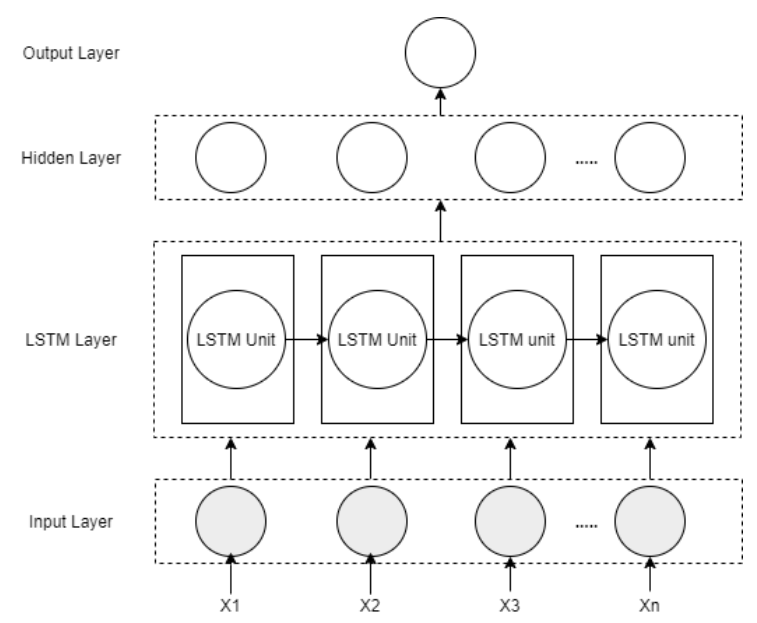

Figure 2. LSTM Network structure

Long short-term memory (LSTM) networks are a modified version of RNNs used in the field of deep learning. Unlike RNN which has a short-term memory, LSTM has memory gates that can learn which word in a sequence of words is important to keep or can be put aside. Figure 2 represents a schematic view of a LSTM neural network. The first layer is the input layer and the number of neurons in this layer is equal to the dense vectors dimension which is encoded by applying word embedding on the user profile text data. The second layer is the hidden layer, which consists of a set of recurrently connected blocks, known as LSTM memory blocks. LSTM transforms the input sequence vectors into a single vector, containing information about the entire sequence. The next layer is a hidden dense layer which works as a fully connected network predicting proper weights for the output layer. The output layer provides us with the education level prediction results. Since predicting the user's level of education is a binary classification problem, there is a single neuron regarding to the output layer.

\section{Dealing with Imbalanced data}

The two education level classes are not evenly distributed because $22 \%$ of the users are classified in the lower education class. Imbalanced classes pose challenges to statistical learning because the classification algorithms will be biased towards predicting the majority class and there are insufficient data to learn the patterns present in the minority class. We addressed this problem by assigning a higher class weight to the minority class (i.e., setting the class weight parameter to "balanced" in the scikit-learn library; Pedregosa et al. 2011). For LSTM, we oversampled the minority class in the training set as a method to deal with class imbalances.

\section{Stratified k-fold cross-validation}

To evaluate the performance of the Naive Bayes and L2 regularized logistic regression models, in terms of how each model generalizes to previously unseen data, we split our dataset into a training and a test set with proportions of 0.75 and 0.25 respectively. A stratified split was used to assure that the distribution of the two education level classes is the same in both the training set and the test set. Stratified k-fold cross-validation was used to find the optimal value for the shrinkage parameter in the training set.

\section{Model evaluation}

We used several measures to evaluate the performance of the binary classification models, based on the proportions of correctly and incorrectly predicted instances in the confusion matrix. Each row of 
the confusion matrix represents the instances in an observed education class while each column represents the instances in the predicted education class.

Table 1. Confusion matrix of observed and predicted classes

\begin{tabular}{|l|l|l|l|}
\cline { 2 - 4 } \multicolumn{2}{l|}{} & $\begin{array}{l}\text { Elementary } \\
\text { education }\end{array}$ & $\begin{array}{l}\text { Higher } \\
\text { education }\end{array}$ \\
\hline \multirow{2}{*}{$\begin{array}{l}\text { Observed } \\
\text { class }\end{array}$} & $\begin{array}{l}\text { Elementary } \\
\text { education }\end{array}$ & TN & FP \\
\cline { 2 - 4 } & $\begin{array}{l}\text { Higher } \\
\text { education }\end{array}$ & FN & TP \\
\hline \multicolumn{2}{|l|}{ Predicted class } \\
\cline { 2 - 4 }
\end{tabular}

- TN (True Negative, True low-education): The number of instances that the classifier has labeled correctly as elementary-education class.

- FN (False Negative, False low-education): The number of instances that actually belong to the higher education class but the classifier has labeled them incorrectly as elementary-education class.

- FP (False Positive, False higher education): The number of instances that actually belong to the elementary-education class but the classifier has labeled them incorrectly as higher education class.

- TP (True Positive, True higher education): The number of instances that the classifier has labeled correctly as higher education class.

The confusion matrix shows the practical relevance of the model. In particular, the successful predictions of the classifier are on the main diagonal where the observed class matches the predicted class. The cells off the diagonal represent misclassified samples. Especially in the presence of class imbalance, the confusion matrix will give insight in the way the observations in the minority class are classified, compared to the majority class. We used the following measures to summarize the most important patterns in the confusion matrix, to be able to evaluate the performance of the classification models.

Precision indicates the fraction of relevant instances (the correctly identified higher educated users) among all positively labeled instances (false positives and true positives).

$$
\text { Precision }=\frac{\text { True Positives }}{\text { True Positives }+ \text { False Positives }}
$$

Recall measures the proportion of positives that are correctly classified (i.e., the percentage of higher educated users that are correctly predicted as higher educated). The F1 score is the harmonic mean of precision and recall that combines both measures as a single number and performs well in the presence of imbalanced classes (Guido, S. and Müller, A., 2016).

$$
\text { Recall }=\frac{\text { True Positives }}{\text { True Positives }+ \text { False Negatives }}
$$




$$
F 1=2 \times \frac{\text { Precision } * \text { Recall }}{\text { Precision }+ \text { Recall }}
$$

Looking only at precision or recall will not provide us with a full picture of model performance. Looking for a system which provides both high precision and high recall requires a trade-off between these two metrics. For each evaluation measure (precision, recall and F1-score) we report the weighted averages over the two education classes, where the weights are equal to the proportion of users in each education class (Müller and Guido 2016).

\section{RESULTS}

Table 2 shows the evaluation measures on the test set for the three classifiers on the simple bag-of-words model (BGW) with lexical features only. L2 regularized logistic regression and LSTM show the best results. Considering that not only the performance but also the interpretability and the simplicity of the model is important, we chose regularized logistic regression as the main classifier algorithm to be used to test the remaining text representation models: the model with the linguistic features only (LWC) and the model with the combination of textual and linguistic features (BLWC).

Table 2. Performance measures for the three classifiers for the bag-of-words model (BGW)

\begin{tabular}{llll}
\hline Measures & Naïve Bayes & $\begin{array}{l}\text { L2 } \\
\text { regularized } \\
\text { Logistic } \\
\text { Regression }\end{array}$ & \\
\hline weighted avg precision & 0.81 & 0.82 & 0.82 \\
\hline weighted avg recall & 0.76 & 0.81 & 0.82 \\
\hline weighted avg F1-score & 0.77 & 0.82 & 0.82 \\
\hline
\end{tabular}

Table 3 displays the results of the prediction of educational level based on three different text representation models (BGW, LWC and BLWC), obtained with $\mathrm{L} 2$ regularized logistic regression. 
Table 3. Model evaluation measures for $L 2$ logistic regression for the different text representation models (BGW, LWC and BLWC)

\begin{tabular}{lllll}
\hline $\begin{array}{l}\text { Model evaluation } \\
\text { measures }\end{array}$ & $\begin{array}{l}\text { Model-1 } \\
\text { BGW }\end{array}$ & $\begin{array}{l}\text { Model-2 } \\
\text { LWC }\end{array}$ & $\begin{array}{l}\text { Model-3 } \\
\text { BLWC }\end{array}$ \\
\hline $\begin{array}{l}\text { Weighted } \\
\text { precision }\end{array}$ & avg & 0.82 & 0.77 & 0.83 \\
\hline weighted avg recall & 0.81 & 0.70 & 0.83 \\
\hline weighted avg F1 & 0.82 & 0.72 & 0.83 \\
\hline
\end{tabular}

The weighted average precision, recall and F1 scores show that the best results are obtained with the BLWC model, the model that combines lexical features with linguistic features. The model with only linguistic features (LWC) clearly performs less well than the other two models. It should be noted that the simple bag-of-words model (BGW) with only textual features performs almost as good as the model combining textual and linguistic features (BLWC). However, the BLWC model provides more informative and interpretable features for each education level class.

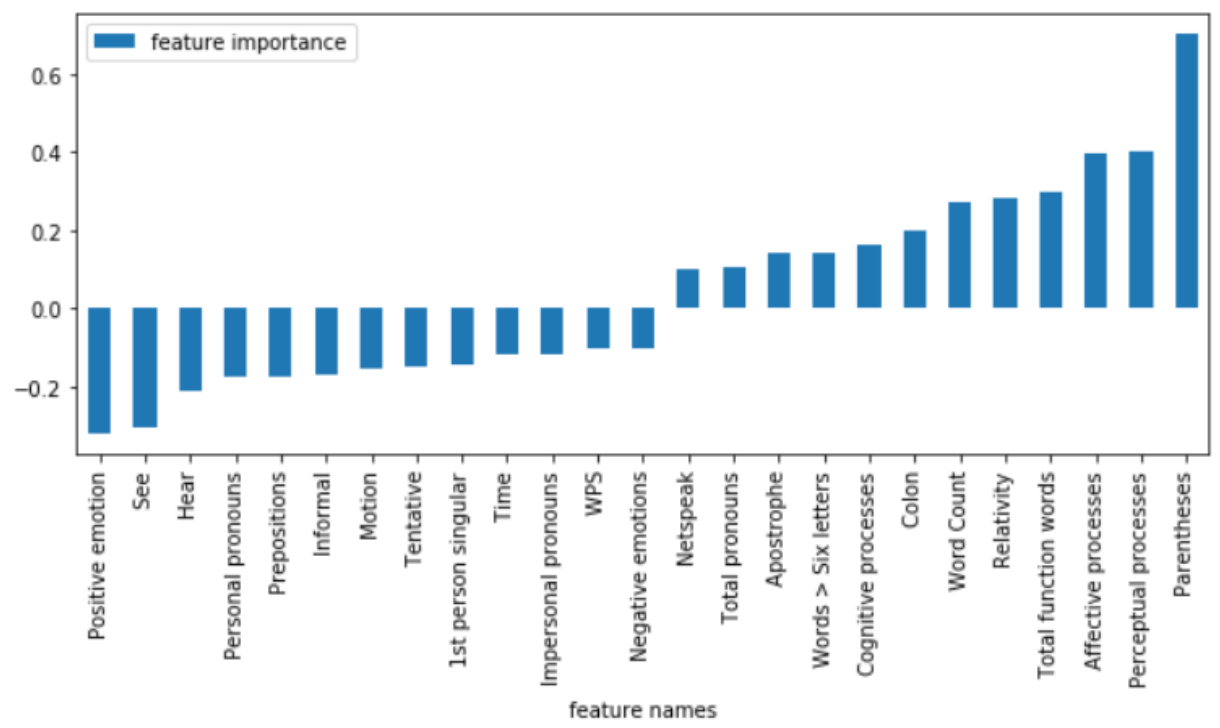

Figure 3. Regression coefficients (y-axis) for the most important textual and linguistic features from the $B L W C$ model obtained with $L 2$ regularized logistic regression.

Figure 3 shows the regression coefficients of the most important lexical and linguistic features of the BLWC model obtained with $L 2$ regularized logistic regression. The negative coefficients on the left side belong to features that are associated with lower education level, while the positive coefficients on the right side are associated with higher education level.

The use of parentheses, and to a lesser extent, colons and apostrophes, are more prominent for users in the higher education category, which suggests that the use of more complex sentence structures are 
indicative of a higher level of education. In addition, the user profiles of the higher educated show more signs of higher levels of language proficiency than the lower educated as indicated by the features "word count", "words longer than six letters" and "cognitive processes". Higher educated users tend to use more words and longer words and their texts show more signs of cognitive complexity. The use of words longer than six letters is associated with higher language fluency (Tausczik and Pennebaker 2010). On the other hand, the use of informal language, which includes subcategories such as swear words, "netspeak" (e.g., "btw", "lol", "thx"), no fluency words ("er", "hm", "umm"), fillers ("Imean", "You know") are prominent features for labeling users in the lower education class, which suggest that higher-educated users are more likely to use a somewhat more formal and polite style. The use of the first person singular and tentative language use are among the most important features in the lower education class. According to Tausczik and Pennebaker (2010:33) "lower-status language is more selffocused and tentative". Among the psychological aspects, we find the use of all the feelings (positive, negative, anxiety, anger and sadness) in the higher education class while for the users in the contrary class only the use of positive and negative emotions is prominent. This finding seems somewhat at odds with the emerging literature on emotion and social class, which suggests that specific positive emotions such as compassion are more prominent among people form lower socioeconomic strata (Piff and Moskowitz 2018). Given the limited contribution of LIWC features in classifying levels of education, however, interpreting such psychological differences in more detail is beyond the scope of this paper.

\section{DISCUSSION}

Overall, we find that we can predict an author's educational background with reasonable accuracy using only the bag-of-words approach. Adding linguistic features (LIWC) to BGW improves accuracy only marginally, while LIWC alone performs clearly worse than BGW. From a substantive point of view, this is perhaps somewhat surprising, as at least intuitively, the language use of the higher educated is distinguished by more than just the use of a certain vocabulary (which is what BGW basically implies, although our use of bigrams implies some context). Also, from a theoretical point of view, this finding at first sight provides little support for the notion that the use of language serves as a form of cultural capital in the context of online dating. After all, as compared to more subtle stylistic features, a vocabulary is relatively easy to learn and as such hardly serves as a credible signal of social background. Nevertheless, we do find that the language usage of highly educated users is empirically distinguishable from that of lower educated users, so even if a "higher educated vocabulary" may be relatively easy to fake, lower educated users don't apparently do this. An exception is in particular the use of parentheses (along with other indicators of complexity), suggesting that higher educated users write in more complex sentences, which is less likely to be mimicked and thereby more likely to be a signal of social background, and in this sense cultural capital. This result also shows that if the use of language is to be related to the characteristics of the author, it may be important to preserve features like punctuation that are typically discarded in preprocessing.

From an empirical perspective, the finding that BGW alone can predict educational background reasonably well, may actually be an advantage, as it implies that looking at longer, more complex structure of sentences does not contribute much. This has two further implications that are of practical value in empirical research. First, if the order of words does not matter, words in user-produced texts may be shuffled to preserve the anonymity of authors. Second, the fact that the more complex structure of sentences contributes little to prediction suggests that words may be sampled, or that shorter texts (as compared to the relatively long essays that we studied in this paper) may suffice, such as the tweets studied in some earlier research. Assessing how our methods performs on shorter samples of texts, as compared to the complete texts, would be a natural next step in our research. 
In closing, we discuss a number of limitations of our study and possible avenues for further research. First, our target variable, level of education, is not without issues. Although the fact that we have userreported level of education may be an advantage over earlier research were background variables often had to be manually annotated based on contextual information, there is no guarantee that the reported level of education is correct. In particular in the "strategic" context of online dating, users may have incentives to overstate their level of education, or, not report it at all (the latter is perhaps more likely given the expected negative consequences of lying about one's credentials in a dating context). In part this may also explain the limited variance in levels of education, which is from an empirical point of view probably the strongest limitation of our data. Apart from biases created by self-reporting, the limited variance may also be due to the context in which the data were collected: not only is the number of formal levels of education limited in United States to start with, but the level of education among users of an online dating platform in the Bay Area can also be expected to be higher than average. Further research may therefore aim to replicate our method using better samples, in particular with verified (or verifiable) educational credentials, more diverse populations, and in different geographic contexts where the variance of education levels may be higher.

Second, more substantively, although our experiments with LIWC do not indicate that looking into more intricate features of language beyond choice of words (BGW) promises strong improvements in prediction, we did not explore other methods of quantifying text in terms of grammar or style (c.f. Macaulay 2005), as we are currently not aware of such methods. Nevertheless, although BGW in combination with LIWC already performs well, there is also still room for improvement, so future research into such more intricate linguistic features may be worthwhile.

The relative simplicity of the BGW approach also means that it is relatively easy to implement, which may facilitate the application of the method also beyond strict text mining context, for example in research context where missing data on social background can be imputed using information from written text. A further extension which is also relatively straightforward in the BGW approach is to apply our method to other languages than English.

\section{ACKNOWLEDGEMENTS}

This research was supported by the Dutch Research Council (NWO) under grant number 452-16-002 and by Utrecht University through an Applied Data Science Research Grant. We thank Marco Spruit and Pablo Mosteiro for helpful suggestions.

\section{REFERENCES}

Blau, Peter M., and Otis Dudley Duncan. 1967. The American Occupational Structure. New York: Wiley.

Botsman, Rachel, and Roo Rogers. 2011. What's Mine Is Yours: How Collaborative Consumption Is Changing the Way We Live. London: Collins.

Bourdieu, Pierre. 1973. "Cultural Reproduction and Social Reproduction." Pp. 71-112 in Knowledge, Education, and Cultural Change: Papers in the Sociology of Education, edited by R. Brown. London: Tavistock Publications.

Bourdieu, Pierre. 1991. Language and Symbolic Power. Cambridge, MA: Harvard University Press. 
Burger, John D., John Henderson, George Kim, and Guido Zarrella. 2011. "Discriminating Gender on Twitter." Pp. 1301-9 in Proceedings of the 2011 Conference on Empirical Methods in Natural Language Processing. Edinburgh, Scotland,: Association for Computational Linguistics.

Coleman, James S. 1990. Foundations of Social Theory. Cambridge, MA: Belknap.

Corten, Rense. 2019. "Social Dilemmas in the Sharing Economy." Pp. 278-89 in Handbook of the Sharing Economy, edited by Russell W. Belk, Giana M. Eckhardt, and Fleura Bardhi. Cheltenham: Edward Elgar.

DiPrete, Thomas A., and Gregory M. Eirich. 2006. "Cumulative Advantage as a Mechanism for Inequality: A Review of Theoretical and Empirical Developments." Annual Review of Sociology 32(1):271-97. doi: 10.1146/annurev.soc.32.061604.123127.

Eisenstein, Jacob. 2019. Introduction to Natural Language Processing (Adaptive Computation and Machine Learning Series). Cambridge, MA: The MIT Press.

Eisenstein, Jacob, Brendan O'Connor, Noah A. Smith, and Eric P. Xing. 2010. "A Latent Variable Model for Geographic Lexical Variation." Pp. 1277-87 in Proceedings of the 2010 Conference on Empirical Methods in Natural Language Processing. MIT, MA: Association for Computational Linguistics.

Evans, James A., and Pedro Aceves. 2016. "Machine Translation: Mining Text for Social Theory." Annual Review of Sociology 42(1):21-50. doi: 10.1146/annurev-soc-081715-074206.

Gottfried, Jeffrey, and Elisa Shearer. 2016. News Use across Social Media Platforms 2016. Pew Research Center.

Hastie, Trevor, Robert Tibshirani, and Jerome Friedman. 2009. The Elements of Statistical Learning: Data Mining, Inference, and Prediction. New York, NY: Springer Science \& Business Media.

Hochreiter, Sepp, and Jürgen Schmidhuber. 1997. "Long Short-Term Memory." Neural Computation 9(8):1735-80.

Hofstra, Bas, and Niek C. de Schipper. 2018. "Predicting Ethnicity with First Names in Online Social Media Networks." Big Data \& Society 5(1):2053951718761141. doi: 10.1177/2053951718761141.

ter Huurne, Maarten, Jonas Moons, Amber Ronteltap, Rense Corten, and Buskens, Vincent. 2017. How Linguistic Features of Seller Profiles in the Sharing Economy Predict Trustworthiness. Utrecht: Utrecht University.

ter Huurne, Maarten, Amber Ronteltap, Rense Corten, and Vincent Buskens. 2017. "Antecedents of Trust in the Sharing Economy: A Systematic Review." Journal of Consumer Behaviour 16(6):48598. doi: 10.1002/cb.1667.

Jurafsky, Daniel, and James H. Martin. 2008. Speech and Language Processing: An Introduction to Speech Recognition, Computational Linguistics and Natural Language Processing. Upper Saddle River, NJ: Prentice Hall.

Keijzer, Marijn, and Rense Corten. 2017. In Status We Trust: A Vignette Experiment on Socioeconomic Status and Reputation Explaining Interpersonal Trust in Peer-to-Peer Markets. SocArXiv. 
Kim, Albert Y., and Adriana Escobedo-Land. 2015. "OkCupid Data for Introductory Statistics and Data Science Courses." Journal of Statistics Education 23(2).

Macaulay, Ronald K. S. 2005. Talk That Counts: Age, Gender, and Social Class Differences in Discourse. New York: Oxford University Press.

Molina, Mario, and Filiz Garib. 2019. "Machine Learning for Sociology." Annual Review of Sociology 45:27-45.

Müller, Andreas C., and Sarah Guido. 2016. Introduction to Machine Learning with Python: A Guide for Data Scientists. Sebastopol, CA: O’Reilly Media.

Nguyen, Dong, Rilana Gravel, Dolf Trieschnigg, and Theo Meder. 2013. "' How Old Do You Think I Am?'; A Study of Language and Age in Twitter." in Proceedings of the seventh international AAAl conference on weblogs and social media. AAAI Press.

Nguyen, Dong, Noah A. Smith, and Carolyn Rose. 2011. "Author Age Prediction from Text Using Linear Regression." Pp. 115-23 in Proceedings of the 5th ACL-HLT workshop on language technology for cultural heritage, social sciences, and humanities.

Pedregosa, Fabian, Gaël Varoquaux, Alexandre Gramfort, Vincent Michel, Bertrand Thirion, Olivier Grisel, Mathieu Blondel, Peter Prettenhofer, Ron Weiss, and Vincent Dubourg. 2011. "ScikitLearn: Machine Learning in Python." The Journal of Machine Learning Research 12:2825-30.

Pennebaker, James W., Ryan L. Boyd, Kayla Jordan, and Kate Blackburn. 2015. The Development and Psychometric Properties of LIWC2015. Austin: University of Texas.

Piff, Paul K., and Jake P. Moskowitz. 2018. "Wealth, Poverty, and Happiness: Social Class Is Differentially Associated with Positive Emotions." Emotion 18(6):902.

Piff, Paul K., Daniel M. Stancato, Stéphane Côté, Rodolfo Mendoza-Denton, and Dacher Keltner. 2012. "Higher Social Class Predicts Increased Unethical Behavior." Proceedings of the National Academy of Sciences of the United States of America 109(11):4086-91. doi: 10.1073/pnas.1118373109.

Rao, Delip, David Yarowsky, Abhishek Shreevats, and Manaswi Gupta. 2010. "Classifying Latent User Attributes in Twitter." P. 37 in Proceedings of the 2nd international workshop on Search and mining user-generated contents - SMUC'10. Toronto, ON, Canada: ACM Press.

Ridgeway, Cecilia L. 2014. "Why Status Matters for Inequality." American Sociological Review 79(1):116.

Salganik, Matthew J. 2018. Bit by Bit. Princeton, NJ: Princeton University Press.

Sauder, Michael, Freda Lynn, and Joel M. Podolny. 2012. "Status: Insights from Organizational Sociology." Annual Review of Sociology 38:267-83.

Schor, Juliet B., Connor Fitzmaurice, Lindsey B. Carfagna, Will Attwood-Charles, and Emilie Dubois Poteat. 2016. "Paradoxes of Openness and Distinction in the Sharing Economy." Poetics 54:6681. 
Sherna, Noah. 2012. "Texting Overtakes Talking as Most Popular Form of Communication in UK." The Independent. Retrieved January 12, 2021 (https://www.independent.co.uk/news/uk/homenews/texting-overtakes-talking-most-popular-form-communication-uk-7956016.html).

Tausczik, Yla R., and James W. Pennebaker. 2010. "The Psychological Meaning of Words: LIWC and Computerized Text Analysis Methods." Journal of Language and Social Psychology 29(1):2454.

UNESCO. 2012. International Standard Classification of Education: ISCED 2011. Montreal: UNESCO Institute for Statistics.

Zuckerman, Arthur. 2020. "35 OkCupid Statistics: 2019/2020 Online Dating, User Trends \& Industry Growth." CompareCamp.Com. Retrieved October 1, 2020 (https://comparecamp.com/okcupid-statistics/). 